\title{
Evaluation of hepcidin as a biomarker for the differential diagnosis of iron deficiency anaemia and anaemia of chronic disease
}

\author{
Ephraim Chikwanda', Victor Daka², Marah Simakando ${ }^{3}$, Sumbukeni Kowa ${ }^{4}$, Trevor Kaile ${ }^{5}$ \\ ${ }^{1}$ Scientific Officer-Microbiology, ${ }^{2}$ Scientific Officer and Quality Officer, Department of Biomedical Sciences, Tropical \\ Diseases Research Centre, Ndola, ${ }^{3}$ Lecturer, Departments of Pathology \& Microbiology, ${ }^{5}$ Professor and Dean, \\ Department of Pathology and Microbiology, School of Medicine, University of Zambia, ${ }^{4}$ Lecturer and Head, Department \\ of Food and Drugs, University Teaching Hospital, Lusaka, Zambia
}

Background: Anaemia affects approximately 1.62 billion people globally corresponding to $24.8 \%$ of the world's population. Iron deficiency anaemia (IDA) and anaemia of chronic disease (ACD) are the most common forms of anaemia. A hormone produced by the liver, hepcidin, is the primary regulator of iron homeostasis and its production increases in ACD and decreases in IDA. Usually, ACD and IDA coexist and sometimes look identical on peripheral blood smears. Aims and Objectives: The current study aims to evaluate the diagnostic value of hepcidin to predict ACD from IDA as well as the diagnostic value of hepcidin to predict ACD from a combination of IDA and ACD. Materials and Methods: Specimens presenting with haematological indices suggestive of IDA and/or ACD following World Health Organisation (WHO) standard case definitions were identified among samples coming to the Haematology laboratory for routine investigations. Serum hepcidin, serum ferritin, serum iron and total iron binding capacity (TIBC) were assessed. Demographic data was obtained from specimen requisition forms. Results: Of the 66 participants, $62.1 \%(n=41)$ were females. IDA was more common among females $(36.4 \%)$ than males $(6.1 \%)$ while ACD was more common in males $(19.7 \%)$ than females (12.1\%). Iron Deficiency Anaemia participants had significantly lower hepcidin levels than ACD $(p<0.001)$. There was a significant positive correlation between serum hepcidin and serum ferritin levels $(p<0.001)$. Conclusion: We found that IDA participants had significantly lower hepcidin levels than ACD and IDA/ACD combined. Therefore, serum hepcidin could be considered in diagnosing and distinguishing ACD from IDA or IDA/ACD as it also had high diagnostic sensitivity and specificity compared to other markers.

Key words: Hepcidin, Iron deficiency anaemia, Anaemia of chronic disease
Access this article online Website: http://nepjol.info/index.php/AJMS DOI: 10.3126/ajms.v9i1.18505 E-ISSN: 2091-0576 P-ISSN: 2467-9100

\section{INTRODUCTION}

Anaemia, a condition resulting from reduced number of red blood cells (RBCs) or their oxygen carrying capacity, affects approximately 1.62 billion people globally corresponding to $24.8 \%$ of the world's population. ${ }^{1}$ Iron deficiency anaemia (IDA) is the most prevalent form of anaemia affecting nearly a billion people worldwide followed by anaemia of chronic disease (ACD), a form of anaemia commonly found among hospitalized patients. ${ }^{2,3}$
Hepcidin, a hormone produced in the liver, encoded in humans by Hepcidin Antimicrobial Peptide (HAMP) gene, is the primary regulator of iron homeostasis. ${ }^{4-6}$ Since its discovery by Krause et al. ${ }^{7}$ and Park et al. ${ }^{8}$ there has been an increased understanding of iron homeostasis and the role of hepcidin in ACD. Recently, several studies have been conducted to evaluate the role of hepcidin in various diseases and its diagnostic reliability in distinguishing IDA from ACD., ${ }^{3,5,9-14}$ The first successful validation of a competitive enzyme-linked immunosorbent assay 
(c-ELISA) for detecting physiological and pathological changes in serum and urine hepcidin was conducted by Ganz et al..$^{15}$ Hepcidin measurement could be helpful in distinguishing ACD from IDA, as hepcidin production differs in these forms anaemia.

The laboratory diagnosis of IDA and ACD is traditionally performed by assessing serum iron, total iron binding capacity (TIBC), transferrin saturation (TSAT), serum ferritin and RBC indices namely haemoglobin $(\mathrm{Hb})$, mean cell haemoglobin $(\mathrm{MCH})$, mean cell haemoglobin concentration (MCHC), mean cell volume (MCV) and red cell distribution width (RDW). Several studies have shown that these parameters have some limitations in distinguishing IDA from ACD especially when they are used individually. 12,16 Moreover, in Zambia, the diagnosis of anaemia is mostly based on RBC indices that have little value in differentiating IDA from $A C D$ as these conditions sometimes show similar changes in RBC indices. Therefore, the objective of this study was to evaluate hepcidin as a diagnostic test for the differential diagnosis of IDA and ACD.

\section{MATERIALS AND METHODS}

This was a Cross Sectional Study undertaken from November 2015 to February 2016 at Ndola Teaching Hospital (NTH) in Ndola, Zambia. Specimens from anaemic individuals aged 18 to 60 years presenting with haematological indices suggestive of IDA and/or ACD as defined by WHO standard case definitions were identified among samples coming to NTH-Haematology laboratory for routine investigations. Blood samples were collected from 66 anaemic individuals. Pregnant women and individuals on estrogen, erythropoietin stimulating agents (ESA) or iron therapy were excluded from the study. Further, patients with thalassemia, sideroblastic anaemia or any other anaemia other than IDA and/or ACD were not included in the study. Serum hepcidin, serum ferritin, serum iron and total iron binding capacity (TIBC) were assessed for each patient. Demographic data was extracted from specimen requisition forms. All participants were only enrolled into the study after providing informed consent.

Data was analyzed using IBM SPSS Statistical package version 21. The independent sample t-test was used to determine differences in means between the study groups. Correlation between variables was calculated using Pearson's correlation analysis. The diagnostic utility of serum hepcidin level as a test for differential diagnosis of IDA and ACD was evaluated according to the area under the receiver operating characteristics (ROC) curve. A p-value less than $5 \%$ was considered for all results. Data were expressed as mean \pm SD unless where stated.
Ethical clearance was obtained from the University of Zambia Biomedical Research Ethics Committee (UNZABREC) before the commencement of the study. Permission to conduct the study at NTH was obtained from NTH management.

\section{RESULTS}

Demographic characteristics of the study population Of the 66 anaemic individuals, 41(62.1\%) were females and $25(37.9 \%)$ were males. Iron deficiency anaemia was more common among females $(36.4 \%[\mathrm{n}=24])$, whereas ACD was more common among males $(19.7 \%[\mathrm{n}=13])$ as shown in Table 1 . We found that $8(12.1 \%)$ males and nine $9(13.6 \%)$ females had a combination of IDA and ACD. The mean age (SD) for the study population was $35.4( \pm 11.9)$ years and IDA participants were younger with mean age (SD) of $26.5( \pm 5.9)$ compared to $41.9( \pm 10.0)$ and 42.3 ( \pm 12.7$)$ in ACD and IDA/ACD patients respectively.

The relationship between haematological and biochemical markers of study participants

Table 2 shows the mean serum levels of haematological and biochemical markers of study participants and Table 3 shows the comparison of the mean serum levels between groups. The study showed that IDA and IDA/ACD participants had significantly lower hepcidin levels than ACD $(p<0.001)$ whereas serum ferritin levels were significantly higher in ACD than in IDA ( $p<0.001)$. We also found a significant difference in CRP levels between IDA and ACD ( $p<0.001)$; IDA and IDA/ACD $(p<0.001)$; and ACD and IDA/ACD ( $p=0.007)$. However, we found no statistically significant difference in mean serum ferritin concentration between IDA/ACD and $\operatorname{ACD}(p=0.072)$.

Correlation between hepcidin and haematological and biochemical parameters of study population

Table 4 shows the relationship between hepcidin and haematological and biochemical markers of anaemia. Serum hepcidin levels correlated slightly better with serum ferritin levels and MCV (Pearson's $\mathrm{r}=0.624$, $\mathrm{p}<0.001$; $\mathrm{r}=0.508, \mathrm{p}<0.001$, respectively) than CRP (Pearson's $\mathrm{r}=0.422, \mathrm{p}<0.001)$. There was a weak negative correlation between serum hepcidin and RDW (Pearson's $\mathrm{r}=-0.279$, $\mathrm{p}=0.024)$. However, there was a statistically insignificant correlation between hepcidin and TIBC, serum iron, $\mathrm{MCHC}, \mathrm{HCT}, \mathrm{Hb}$ and RBC.

Diagnostic characteristics for the detection of iron deficiency anaemia and anaemia of chronic disease The Receiver operating characteristics (ROC) curves shown in Figure 1 was used to test the markers' diagnostic performance in IDA and ACD. In IDA, ferritin exhibited

Asian Journal of Medical Sciences | Jan-Feb 2018 | Vol 9 | Issue 1 
Table 1: Demographic characteristics of study participants by sex and age

\begin{tabular}{lcccc} 
& IDA & ACD & IDA/ACD & Total \\
\hline Number cases & $28(42.4 \%)$ & $21(31.8 \%)$ & $17(25.8 \%)$ & 66 \\
Male & $4(6.1 \%)$ & $13(19.7 \%)$ & $8(12.1 \%)$ & $25(37.9 \%)$ \\
Female & $24(36.4 \%)$ & $8(12.1 \%)$ & $9(13.6 \%)$ & $41(62.1 \%)$ \\
Mean age (SD) & $26.5(5.9)$ & $41.9(10.0)$ & $42.3(12.7)$ & $35.4(11.9)$ \\
\hline
\end{tabular}

\section{Table 2: Levels haematological and biochemical indices in IDA, ACD and IDA/ACD}

\begin{tabular}{|c|c|c|c|}
\hline & IDA $n=28$ & ACD $n=21$ & IDA/ACD $n=17$ \\
\hline $\mathrm{RBC}\left(10^{\wedge} 12 / \mathrm{L}\right)$ & $3.10 \pm 1.02$ & $4.05 \pm 5.93$ & $3.37 \pm 0.69$ \\
\hline $\mathrm{Hb}(\mathrm{g} / \mathrm{dL})$ & $6.5 \pm 2.5$ & $8.1 \pm 1.9$ & $7.4 \pm 1.8$ \\
\hline HCT $(\%)$ & $21.1 \pm 7.4$ & $25.3 \pm 5.1$ & $25.8 \pm 7.1$ \\
\hline $\mathrm{MCV}(\mathrm{fL})$ & $65.1 \pm 6.9$ & $73.8 \pm 3.2$ & $69.1 \pm 5.6$ \\
\hline $\mathrm{MCHC}(\mathrm{g} / \mathrm{dL})$ & $30.8 \pm 2.8$ & $31.8 \pm 2.52$ & $31.2 \pm 2.7$ \\
\hline RDW (\%) & $21.8 \pm 5.1$ & $17.3 \pm 1.8$ & $20.7 \pm 3.6$ \\
\hline Serum iron $(\mu \mathrm{g} / \mathrm{dL})$ & $36.85 \pm 12.01$ & $42.14 \pm 12.61$ & $41.88 \pm 13.48$ \\
\hline TSAT $(\%)$ & $8.02 \pm 3.03$ & $7.73 \pm 2.87$ & $9.68 \pm 2.47$ \\
\hline $\mathrm{CRP}(\mathrm{mg} / \mathrm{L})$ & $5.03 \pm 6.69$ & $30.60 \pm 8.28$ & $22.14 \pm 9.91$ \\
\hline Ferritin (ng/mL) & $10.87 \pm 7.76$ & $118.76 \pm 42.28$ & $63.60 \pm 22.9$ \\
\hline Hepcidin (ng/mL) & $6.41 \pm 2.21$ & $15.39 \pm 4.70$ & $7.31 \pm 2.01$ \\
\hline
\end{tabular}

\begin{tabular}{|c|c|c|c|c|c|c|}
\hline & \multicolumn{2}{|c|}{ IDA vs ACD } & \multicolumn{2}{|c|}{ IDA vs IDA/ACD } & \multicolumn{2}{|c|}{$A C D$ vs IDA/ACD } \\
\hline & t-test & p-value & t-test & p-value & t-test & $p$-value \\
\hline Age (years) & -6.768 & $<0.001$ & -5.868 & $<0.001$ & -0.111 & 0.912 \\
\hline $\operatorname{RBC}\left(10^{\wedge} 12 / L\right)$ & 2.645 & 0.011 & 2.797 & 0.008 & 0.844 & 0.404 \\
\hline $\mathrm{Hb}(\mathrm{g} / \mathrm{dL})$ & -1.171 & 0.247 & 1.404 & 0.168 & 2.16 & 0.038 \\
\hline HCT $(\%)$ & 0.264 & 0.793 & 2.139 & 0.038 & 2.087 & 0.044 \\
\hline $\operatorname{MCV}(f L)$ & -5.398 & $<0.001$ & -2.005 & 0.051 & 3.294 & 0.002 \\
\hline $\mathrm{MCHC}(\mathrm{g} / \mathrm{dL})$ & -1.328 & 0.191 & -0.499 & 0.62 & 0.709 & 0.483 \\
\hline RDW (\%) & 3.928 & $<0.001$ & -0.88 & 0.384 & -3.731 & 0.001 \\
\hline Serum Fe $(\mu \mathrm{g} / \mathrm{dL})$ & -1.495 & 0.142 & -1.302 & 0.2 & 0.61 & 0.952 \\
\hline TIBC $(\mu \mathrm{g} / \mathrm{dL})$ & -0.379 & 0.706 & -0.327 & 0.746 & 0.053 & 0.958 \\
\hline TSAT $(\%)$ & -1.997 & 0.052 & -1.897 & 0.065 & 0.063 & 0.95 \\
\hline CRP (mg/L) & -11.954 & $<0.001$ & -6.918 & $<0.001$ & 2.866 & 0.007 \\
\hline Ferritin (ng/mL) & -8.799 & $<0.001$ & -8.999 & $<0.001$ & 1.782 & 0.072 \\
\hline Hepcidin (ng/mL) & -6.27 & $<0.001$ & -2.307 & 0.026 & 4.356 & $<0.001$ \\
\hline
\end{tabular}

The mean difference is statistically significant if $\mathrm{p}<0.05$. CRP=C-reactive protein; TSAT=Transferrin saturation; TIBC=Total iron bind capacity; RDW=Red cell distribution width; $\mathrm{MCHC}=$ Mean cell haemoglobin concentration; $\mathrm{MCV}=$ Mean corpuscular volume; $\mathrm{HCT}=$ Haematocrit; $\mathrm{Hb}=$ Haemoglobin; RDW=Red blood cell

\begin{tabular}{|c|c|c|}
\hline Biomarker & Pearson correlation $(r)$ & $p$-value \\
\hline Ferritin & 0.624 & $<0.001$ \\
\hline CRP & 0.422 & $<0.001$ \\
\hline TSAT & 0.163 & 0.191 \\
\hline TIBC & -0.097 & 0.439 \\
\hline Serum iron & 0.059 & 0.639 \\
\hline RDW & -0.279 & 0.024 \\
\hline $\mathrm{MCHC}$ & -0.034 & 0.789 \\
\hline MCV & 0.508 & $<0.001$ \\
\hline $\mathrm{HCT}$ & 0.065 & 0.602 \\
\hline $\mathrm{Hb}$ & 0.12 & 0.337 \\
\hline RBC & -0.15 & 0.231 \\
\hline
\end{tabular}

Asian Journal of Medical Sciences | Jan-Feb 2018 | Vol 9 | Issue 1 the best performance with $99.5 \%$ area under the curve (AUC) (95\% CI: 98.5 to 100), $96.4 \%$ sensitivity and $73.8 \%$ specificity at $\leq 30 \mathrm{ng} / \mathrm{mL}$ cutoff value whereas hepcidin showed the weakest performance with $85.4 \%$ AUC (95\% CI: 76.4 to 94.3 ), $89.3 \%$ sensitivity and $34.2 \%$ specificity at $\leq 5 \mathrm{ng} / \mathrm{mL}$ cutoff value. In ACD, however, hepcidin showed the best performance with 94.2\% AUC (95\% CI: 88.5 to 99.9 ), $95.2 \%$ sensitivity and $63.8 \%$ specificity compared to ferritin with $86.8 \%$ AUC (95\% CI: 78.5 to 95.1$), 94.1 \%$ sensitivity and $42.9 \%$ specificity, and CRP with 90.5\% AUC (95\% CI: 83.0 to 98.0), $90.2 \%$ sensitivity and $35.2 \%$ specificity. For all the markers, the AUC was significantly different from 0.5 since $\mathrm{p}$-value $<0.001$. 


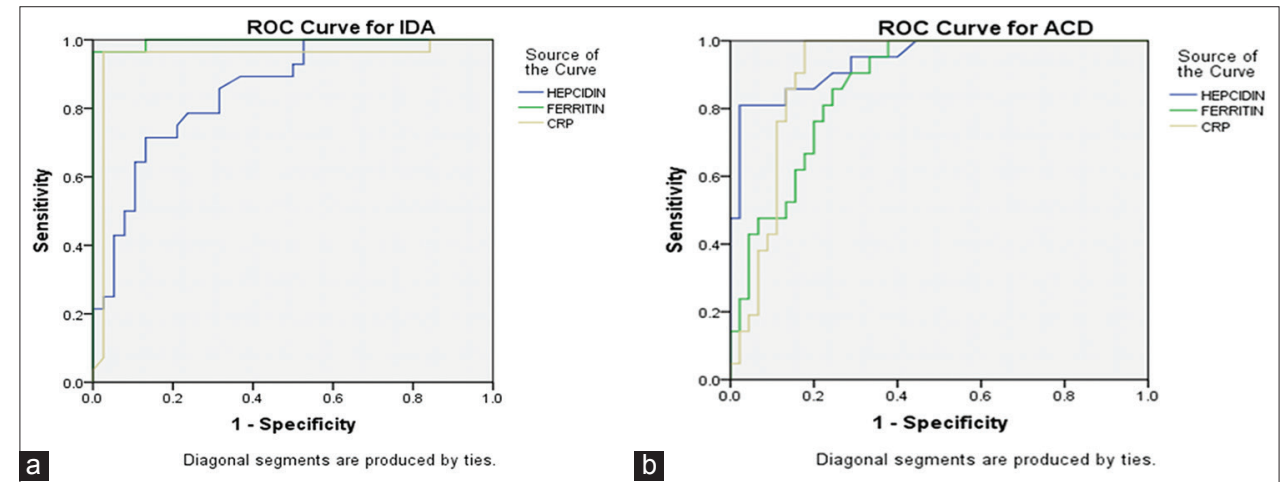

Figure 1: Receiver operating characteristic (ROC) curves for iron deficiency anaemia (a) and anaemia of chronic disease (b). True area=0.5

\section{DISCUSSION}

We found more females $41(62.1 \%)$ with anaemia than males $25(37.9 \%)$ and IDA was the most prevalent form of anaemia accounting for $28(42.4 \%)$ cases of whom $24(36.4 \%)$ were females. Iron deficiency anaemia causes approximately half of all anaemia cases worldwide, and affects females of reproductive age more often than males. Many studies have found that anaemia is a common problem in women of reproductive age resulting from prolonged negative iron balance, caused by inadequate iron intake, or absorption, increased iron demand during pregnancy, and increased iron losses due to menstruation. ${ }^{1,3,16}$ Anaemia of chronic disease, however, was more common among males 13 (19.7\%) and accounted for 21 (31.8\%). The mean age of IDA participant (26.5 years) was significantly lower than ACD (41.9 years) or IDA/ACD (42.3 years), $\mathrm{p}<0.001$. Many studies have shown that ACD is more common in elderly, which is a consequence of the presence of more chronic diseases, rather than a normal aging phenomenon. ${ }^{17}$

The study showed that IDA and IDA/ACD participants had significantly lower hepcidin levels than ACD, but the median serum hepcidin levels between IDA and IDA/ACD was negligible. ACD participants showed a wide range of distribution of hepcidin levels compared to IDA and IDA/ ACD.This is in agreement with the study done by Van Santen ${ }^{18}$ which found that hepcidin content in serum from patients in the IDA group and as well as that from patients combined IDA/ACD group differed significantly from that in the ACD group $(\mathrm{p}<0.001)$. Another study done by Röhrig et al.,showed a statistical significant difference of hepcidin levels among IDA, ACD and control groups $(p=0.034)$.

A strong positive correlation between serum hepcidin levels and serum ferritin levels (Pearson's $r=0.624$, $\mathrm{p}<0.001)$ was also observed. A study done by Röhrig et al., 2014 showed a strong positive correlation between serum hepcidin and ferritin (spearman $\mathrm{r}=0.747) .{ }^{19}$ Serum hepcidin levels also significantly correlated with CRP (Pearson's $r=0.508, p<0.0001$ ). Serum hepcidin, serum ferritin and CRP are induced by inflammation and therefore high levels of these markers are seen in ACD compared to IDA. ${ }^{20}$

The receiver operating characteristic analysis was used to assess the characteristic of hepcidin, ferritin and CRP in IDA and ACD. In IDA, ferritin exhibited the best performance with 99.5\% AUC (95\% CI: 98.5 to 100), $96.4 \%$ sensitivity and $73.8 \%$ specificity at $\leq 30 \mathrm{ng} / \mathrm{mL}$ cutoff value, whereas, in ACD, hepcidin showed the best performance with $94.2 \%$ AUC (95\% CI: 88.5 to 99.9), $95.2 \%$ sensitivity and $63.8 \%$. A study done by Choi et al., 2012, showed that the AUC for serum hepcidin as predictor of iron deficiency (ID) was $85.2 \%$ (95\% CI: 75.5 to 95.0$)$ and hepcidin $\leq 6.895 \mathrm{ng} / \mathrm{mL}$ had a sensitivity of $79.2 \%$ and specificity of $82.8 \%$ for the diagnosis of ID whereas hepcidin $\leq 2.735 \mathrm{ng} / \mathrm{mL}$ had a sensitivity of $88.1 \%$ and specificity of $88.2 \%$ for diagnosis IDA. On the contrary, a study done by Parischa et al., 2010, showed that an undetectable hepcidin $(<5.4 \mathrm{ng} / \mathrm{mL})$ had a sensitivity and specificity of $41.5 \%$ and $97.5 \%$ respectively, and hepcidin $<20 \mathrm{ng} / \mathrm{mL}$ had a sensitivity and specificity of $74.6 \%$ and $83.2 \%$ respectively. ${ }^{21}$ In a study done by Svenson and colleagues in 2015, hepcidin cutoff point value of $8 \mathrm{ng} / \mathrm{mL}$ had a sensitivity of $73 \%$ and specificity of $72 \%$ in identifying iron deficiency anaemia. ${ }^{22}$

There was a statistically significant correlation between hepcidin and MCV and RDW, and a statistically insignificant correlation between hepcidin and $\mathrm{Hb}$ and RBC. Contrary the study done by Mohammad et al., $2013,{ }^{23}$ showed an insignificant correlation between hepcidin and MCV and RDW ( $\mathrm{r}=0.030, \mathrm{p}=0.706$ and $\mathrm{r}=0.106, \mathrm{p}=184$, respectively) and a statistically significant positive correlation between hepcidin and RBCs and $\mathrm{Hb}$. Decreased values of $\mathrm{Hb}, \mathrm{HCT}, \mathrm{MCHC}$, and MCV were seen in both IDA and ACD, whereas serum hepcidin 
levels were lower in IDA than ACD. Therefore, complete blood count indices may not have a linear relationship with hepcidin.

The study further showed that participants with IDA could be differentiated from those with ACD since there was a significant difference in the mean serum hepcidin levels $(\mathrm{p}<0.001)$. In addition, serum hepcidin performed well as a diagnostic test of deficiency of iron, even in the presence of inflammation and differentiated groups with IDA and IDA/ACD ( $p=0.026)$. Both IDA and IDA/ACD had lower hepcidin levels than a pure case of ACD. These findings are consistent with most of the studies that have evaluated the properties of hepcidin as a diagnostic test for differential diagnosis of IDA and ACD. ${ }^{18-22,24,25}$

\section{Limitations}

Although there was a significant difference in serum hepcidin levels between IDA and ACD, serum hepcidin levels could have been done in the normal population to determine reference values.

\section{CONCLUSION}

The study found that IDA participants had significantly lower hepcidin levels than ACD and IDA/ACD participants. It was further discovered that serum hepcidin could be used as one of the iron indices especially in the differentiation of ACD from IDA and IDA/ACD as these forms of anaemia require different approaches to treatment. Therefore, serum hepcidin could be considered in diagnosing and distinguishing ACD from IDA or IDA/ ACD as it had high diagnostic sensitivity and specificity compared to others.

\section{ACKNOWLEDGEMENT}

The lecturers of the University of Zambia, School of Medicine, and Ndola Teaching Hospital Management and Laboratory staff.

\section{REFERENCES}

1. Benoist Bd, McLean E, Egli I, Cogswell M. Worldwide prevalence of anaemia 1993-2005. 2008.

2. Cullis J. Anaemia of chronic disease. (Royal College of Physicians) 2013;13(2):193-196.

3. Ganz T. Hepcidin and iron regulation, 10 years later Blood. 2011;117:4425-4433

4. Basseri RJ, Nemeth E, Vassilaki ME, Basseri B, Enayati $P$, Shaye $\mathrm{O}$, et al. Hepcidin is a key mediator of anemia of inflammation in Crohn's disease. J Crohns Colitis 2013;7(8):e286-e291.
5. De Domenico I, Ward DM and Kaplan J. Hepcidin and ferroportin: the new players in iron metabolism. Semin Liver Dis 2011;31(3):272-279.

6. Ganz T. Hepcidin--a peptide hormone at the interface of innate immunity and iron metabolism. Curr Top Microbiol Immunol 2006;306:183-198.

7. Krause A, Neitz S, Magert HJ, Schulz A and Forssmann WG. LEAP-1, a novel highly disulfide-bonded human peptide, exhibits antimicrobial activity 2000;480:147-150.

8. Park $\mathrm{CH}$, Valore EV, Waring A and Ganz T. Hepcidin, a urinary antimicrobial peptide synthesized in the liver. The Journal of Biological Chemistry 2001;276:7806-7810.

9. Abdel-Khalek MA, El-Barbary AM, Essa SA and Ghobashi AS. Serum hepcidin: a direct link between anemia of inflammation and coronary artery atherosclerosis in patients with rheumatoid arthritis. J Rheumatol 2011;38(10):2153-2159.

10. Antunes SA and Canziani ME. Hepcidin: an important iron metabolism regulator in chronic kidney disease. J Bras Nefrol 2016;38(3):351-355

11. Bergamaschi $G$ and Villani L. Serum hepcidin: a novel diagnostic tool in disorders of iron metabolism. Haematologica 2009;94(12):1631-1633.

12. Brugnara C. Iron deficiency and erythropoiesis: new diagnostic approaches. Clinical Chemistry. Clinical Journal of the American Society of Nephrology 2003;10:1573-1578.

13. Franchini M, Montagnana M and Lippi G. Hepcidin and iron metabolism: from laboratory to clinical implications. Clin Chim Acta 2010;411(21-22):1565-1569.

14. Girelli D, Nemeth E and Swinkels DW. Hepcidin in the diagnosis of iron disorders. Blood 2016;127(23):2809-2813.

15. Ganz T, Olbina G, Girelli D, Meneth E and Westerman M. Immunoassay for human serum hepcidin. Blood 2008;112(10):4292-4297.

16. Wish B. Assessing iron status: beyond serum ferritin and transferritin saturation. Clinical Journal of the American Society of Nephrology 2006;1:S4-S8.

17. Weiss $G$ and Goodnough LT. Anaemia of Chronic Disease. The New England Journal of Medicine 2005;352(10):1011-1023.

18. van Santen $S$, van Dongen-Lases EC, de Vegt F, Laarakkers CM, van Riel PL, van Ede AE, et al. Hepcidin and hemoglobin Content parameters in the diagnosis of iron deficiency in rheumatoid arthritis patients with anaemia. Arthritis Rheum 2011; 63(12):3672-3680.

19. Röhrig G, G Rappl B, Vahldick B, Kaul I and Schulz R. Serum hepcidin levels in geriatric patients with iron deficiency anemia or anemia of chronic disease. Zeitschrift fur Gerontologie und Geriatrie 2014;47(1):51-56.

20. Nemeth E, Rivera S, Gabayan V, Keller C, Taudorf S, Pedersen BK and Ganz T. IL-6 mediates hypoferremia of inflammation by inducing the synthesis of iron regulatory hormone hepcidin. J Clin Invest 2004;113:1271-1276.

21. Parischa SR, McQuilten Z and Westerman M. Serum hepcidin as a diagnostic test of iron deficiency in premenopausal female blood donors. Haematologica 2011;96:1099-1105.

22. Svenson $\mathrm{N}$, Patmore $\mathrm{R}, \mathrm{Cox} \mathrm{H}$, Bailey $\mathrm{J}$ and Holding S. Iron Age or New Age: Ironing out the Diagnosis of Anaemia of Inflammation from Iron Deficiency Anaemia. Hull and East Yorkshire Hospitals NHS Trust. 2015.

23. Mohammad BA, Shubair EM and Zaida MT. Hepcidin Status Correlated with Biochemical Parameters and Hematological Indices among Iron Deficiency Anemic Children aged (6-12) years in Gaza City: A Case Control Study. Journal of Natural and Engineering Studies 2014;22(2):1-13.

24. Choi S, Hyoungi H, Sang S, Hee LJ, Heep-Jin K and Ram YH. 
Serum hepcidin levels and iron parameters in children with iron deficiency. Korean Journey of Hematology 2012;47(4):286-292.

25. Manolov V, Paskaleva-Peycheva V, Bogov B, Yonova D,
Vazelov E, Hadjiev E, et al. Serum hepcidin quantification in differentiation of anemia. International Journal of development research 2015;5(1):2918-2920.

\section{Authors Contribution:}

EC- Project concept and design, data collection, manuscript preparation and revision, statistical data analysis and interpretation; VD-Manuscript preparation and statistically analyzed and interpreted data; MS-Thesis and manuscript editing; SK-Co-supervised the design of the project, data collection, analysis and interpretation, thesis and manuscript editing; TK-Supervised the development of the project, data collection, analysis and interpretation, thesis and manuscript editing..

\section{Work attributed to:}

Department of Pathology and Microbiology, University of Zambia and Ndola Teaching Hospital

\section{Orcid ID:}

Mr. Ephraim Chikwanda:: (i) http://orcid.org/0000-0001-5989-9477

Mr. Victor Daka: (1) http://orcid.org/0000-0001-9490-5609

Ms. Marah Simakando: (1) http://orcid.org/0000-0001-9940-6972

Dr. Simbukeni Kowa: (1) http://orcid.org/0000-0003-2690-2566

Source of Support: Nil, Conflict of Interest: None declared. 